\title{
Preface for a Special Issue on Green Manufacturing and Applications
}

As oil prices escalate, green manufacturing is becoming an increasingly important area of research to implement and achieve sustainable development of local and global economies. This special issue is the first attempt by IJPEM (International Journal of Precision Engineering and Manufacturing) that addresses these concerns from various multidisciplinary fields of green manufacturing as an integrated form. Selected papers from the International Symposium of Green Manufacturing and Applications (ISGMA) 2011, held at Seoul National University, Korea, from October 4 to 6, 2011 and regularly submitted papers in green manufacturing area were compiled to develop this special issue.

The topics cover the design of greener products, bio-based materials, energy saving in various manufacturing processes, fundamental research on renewable energy systems such as fuel cell, wind power system, etc, reviews on green manufacturing and applications. Research efforts in design-manufacturing-materials synergistically provide insights from different viewpoints and offer opportunities to overview state-of-the-art technologies in closely related topics.

I express my deepest gratitude to the organizers of ISGMA 2011 and editorial members of IJPEM as well as authors for their support and contribution to this special issue. Especially, invaluable guidance of general chair, Prof. Chong Nam Chu, and cochairs, Prof. David Dornfeld, Prof. Ichiro Inasaki, Prof. Jon-Won Kim, Dr. Gyu-Bong Lee, and Prof. Friedrich B. Prinz are greatly acknowledged. In addition, special cooperation between the editorial staff of the Korean Society for Precision Engineering and Springer for their hard work and timely response to allow publication of this issue is highly appreciated.

Guest editor: Prof. Sung-Hoon Ahn

School of Mechanical and Aerospace Engineering

Seoul National University 